\title{
Oil Purification Devices Used in Internal Combustion Engines
}

\author{
Shavkat Imomov, Nargiza Kholikova, Zebo Alimova, Ikrom Nuritov, Nargiza Temirkulova
}

\begin{abstract}
The article presents, developed operating modes and substantiates the parameters of a device that cleans motor oils from oxidation products and the resulting analytical relationships representing the movement of a mixture of acetone and used motor oil in viscosity states and the process of evaporation of the solvent from the oil during processing. It also substantiates the speed of movement of motor oil on a coneshaped surface based on its effect on refining and substantiates the dependence of the quality of refined oil on the temperature indicator of the mixture and the availability of the evaporation process and it was also found that motor oils are intensively contaminated with oxidation products during processing, thereby accelerating the friction process. During agricultural work, tractor engines due to congestion and oxidation of carbon, the content of carbine, carbide and asphalt products increases by 1.2\%. Oxidizing products accelerate the deterioration of the quality of oils, which leads to an increase in the power consumption of the engine KShM and CPG by 15\%. In addition, analytical relationships were obtained representing the movement of a mixture of acetone and used motor oil in viscosity states and the process of evaporation of the solvent from the oil during processing. The speed of motor oil on a cone-shaped surface is substantiated based on its effect on refining, and the dependence of the quality of refined oil on the temperature of the mixture and the availability of the evaporation process is justified
\end{abstract}

Keywords: Technology, method, oils, environmental protection, regeneration, cleaning, problem, disposal, membrane, filter, oil collection.

\section{INTRODUCTION}

As is known in world practice, more than 50 million tons of technical lubricants are produced per year, much attention is paid to the cleaning of used oil and bringing it into good condition using various technologies and methods. The development in this area of energy-resource-saving devices that ensure the cleaning of used oils and the production of high-quality oils for use in agricultural technical equipment, another - increasingly exacerbating - environmental protection, which also requires intensive and rational processing of waste hydrocarbon lubricants.

In connection with the above, the development of technologies and devices that increase the resources of used technical oils occupies a leading place. The effective

Revised Manuscript Received on October 15, 2019.

Shavkat Imomov, Tashkent Institute of Irrigation and Agricultural Mechanization Engineers, Tashkent, Uzbekistan. (Email: shavkatimomov@rambler.ru)

Nargiza Kholikova, Tashkent Institute of Irrigation and Agricultural Mechanization Engineers, Tashkent, Uzbekistan. (Email: narbun1977@mail.ru)

Zebo Alimova, The Tashkent institute of projection, building and maintenance of automobile roads, Tashkent, Uzbekistan.

Ikrom Nuritov, Tashkent Institute of Irrigation and Agricultural Mechanization Engineers, Tashkent, Uzbekistan. (Email: ikromnuritov@rambler.ru)

Nargiza Temirkulova, Tashkent state agrarian university, Tashkent, Uzbekistan. organization of the processing of used technical oils, an average of 15 million tons per year, as well as their use in agricultural and reclamation techniques is widespread in world practice [1].

In recent years, a new generation of targeted research has been developed and is being implemented to improve resource-saving devices and technologies that perform the cleaning process of used oils. In this area, including the implementation of the development of a perfect design scheme, devices used to clean used oils, oil purification from oxidation products and obtaining high-quality processed oil, a temperature indicator for the mixture, as well as the evaporation process based on resource saving, is of particular importance.

In the Republic of Uzbekistan, large-scale measures are being taken to restore and cleanse the oils used in agricultural production, to increase the quality of oils used in agricultural machinery and technology. The strategy of actions for the further development of the Republic of Uzbekistan for 2017 - 2021 also includes tasks on "Deepening structural changes and the consistent development of agricultural production, expanding the production of clean products for modernization and accelerated development of agriculture in the priority areas of social development" [2] . To fulfill these tasks, including the development of methods for producing high-quality oil through technical and technological modernization of devices, removal of gaseous products under reduced pressure, qualitatively cleaning used oils from mechanical mixtures, water, organic substances, and oxidized products, it is considered one of the main tasks world community $[3,4,5]$.

In world practice, various devices have been developed for the effective recovery process of used oils. They are recommended by the design bureau and design institutes to create new devices. Hedayatipour M., Jaafarzadeh N., Ahmadmoazzam M. on membrane nanofiltration processes with an acceptable degree of extraction are showing a number of studies on the creation of devices for cleaning used oils, substantiating technological processes and parameters, showing good results in wastewater treatment water; Fu, L., Wu, C., Zhou, Y., Zuo, J., Ding, Y. We studied the parameters of the criteria for evaluating the effect of air-water backwashing on an experimental biological aerated filter that cleans petrochemical contaminated waste. Zhang, J., Qi, J., Shuai, S.-J., Wang, L., Liu, S., Wang, G., Liu, F., Brown, J. Sized diesel particulate filters, which may be reduced by approximately $6 \%$ by

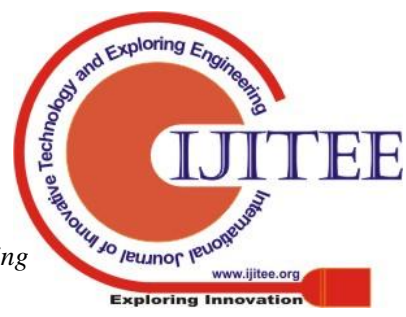


reducing the ash content in lubricating oil from $1.0 \%$ to $0.75 \%$ and by $14 \%$ by reducing from $1.0 \%$ to $0.5 \%$. [5,6,7]. I. Glover, H.J. Jones, A.G. Isah and others were engaged in devices created as a result of these studies, used in agriculture, and to this extent, positive results were achieved. And also in these works, research was conducted on technology and technical means for cleaning used oil, as well as on improving the quality of oils.

However, in these above studies, the tasks of developing a device that completely cleans used oils and substantiates the parameters of working parts ensuring its high quality are not adequately studied.

\section{METHOD}

In the research process, methods of theoretical mechanics, mathematical analysis and mathematical statistics, comparative comparison, generalization and testing methods of agricultural machinery were applied. The main part. It is known that the conditions for the complete cleaning of engine oils from organic pollution are determined by the degree of transparency and reduction of turbidity. Naturally, the diffusion process occurs when motor oils are completely purified from oxidation products.

One of the important tasks is to accurately determine in what proportions motor oils are completely saturated with acetone. During the construction of the corresponding mathematical model, the movement of oils in a fairly concentrated medium between solid particles is replaced by a gross flow, which fills the entire space, which allows us to study the process in the framework of the gross medium.

Considering the normal collision of particles, we describe the speeds of acetone and motor oils $\vec{V}_{a}$ and, $\vec{V}_{\mathcal{M}}$ the particle masses ma and $\mathrm{mm}$ as follows:

$$
\begin{aligned}
& \vec{V}_{a}=V_{a} \cos \alpha \pi \vec{i}+V_{a} \sin \alpha \pi \vec{j} \\
& \vec{V}_{M}=V_{M} \cos \alpha \pi \vec{i}-V_{M} \sin \alpha \overrightarrow{\pi j} \\
& \vec{V}_{a}=V_{a} \sin k \pi \vec{i}-V_{M} \cos k \vec{j} \vec{j}
\end{aligned}
$$

Where: $\vec{V}_{a}$ - is the particle velocity of acetone, $\mathrm{m} / \mathrm{s}$; $\vec{V}_{M}$ are unit vectors, $\mathrm{s} / \mathrm{m}$.

Considering the shape of infinitesimal particles in the form of spheres of radius $\mathrm{Ra}$ and $\mathrm{Rm}$ of densities $\rho \mathrm{a}$ and $\rho \mathrm{m}$, the mass of acetone and motor oil is as follows:

$$
\begin{aligned}
& m_{a}=\frac{4 \pi}{3} \rho_{a} R_{a}^{3} \\
& m_{M}=\frac{4 \pi}{3} \rho_{M} R_{M}^{3}
\end{aligned}
$$

Where; $\mathrm{ma}$ - is the mass of particles of acetone, $\mathrm{kg}$; $\mathrm{mm}$ is the mass of particles of motor oil, $\mathrm{kg}$; $\rho \mathrm{a}$ - the density of particles of acetone; $\rho \mathrm{M}$ - particle density of motor oil; Ra radius of acetone particles; $\mathrm{RM}$-radius of engine oil particles.

Given that the interaction between each liquid and air particles does not affect the collision of adjacent particles, the following expression is given for the ùa velocity vector of the liquid particle:

$$
\vec{u}_{a}=\frac{\left(1-\kappa \frac{m_{a}}{m_{M}}\right) V_{M}+(1+\kappa) \frac{m_{a}}{m_{M}} \vec{V}_{a}}{1+\frac{m_{a}}{m_{M}}}
$$

Where: $\overline{\mathrm{u}}$ - is the mixture velocity parallel to the $\mathrm{x}$ axis, $\mathrm{m} / \mathrm{s} ; \mathrm{k} \quad$-is the coefficient of absorption of acetone and engine oil, $\mathrm{s} / \mathrm{m}$.

Calculation of the velocity of the fluid flow rate:

$$
V=2 \sqrt{\frac{\omega^{2} r \delta(r)}{\chi^{2}}}=2 \sqrt{\frac{\omega^{2} r}{\chi^{2}}(\delta(r)-z)}
$$

Where: $\mathrm{V}$ - is the speed of the mixture parallel to the axis $\mathrm{y}, \mathrm{m} / \mathrm{s} ; \omega-$ is the angular velocity of the growing particles of the mixture, $\mathrm{m} / \mathrm{s} ; \delta(\mathrm{r})$ is the thickness of the boundary layer, $\mathrm{cm}$.

The second flow rate in the boundary zone with thickness $\delta(\mathrm{r})$ is determined from the following equation:

$$
Q=2 \pi r \int_{0}^{\delta(r)} V d z
$$

Where: $\mathrm{Q}$ - is the flow rate of the mixture; $2 \pi \mathrm{r}$ - is the circumference; $\mathrm{V}$ - is the speed of the mixture, $\mathrm{m} / \mathrm{s} ; \delta(\mathrm{r})$ - is the thickness of the boundary layer, sm.

Using expressions (4) and (5), we find the flow rate of the liquid mixture on the film:

$$
\begin{aligned}
& Q=\frac{2 l \pi}{3} \sqrt{\frac{\omega^{2}(r \delta(r))^{3 / 2}}{\chi^{2}}}=\frac{2 \pi l}{3} \sqrt{\frac{\omega^{2} R_{0}^{6}}{\chi^{2}}} \\
& Q_{\text {ж }}=\frac{2 \pi l \omega R_{0}^{3}}{3 \chi} \sqrt{r^{3} \delta^{3}(r)} \\
& Q=\left(\frac{3 Q}{2 \pi l \omega R_{0}^{3}}\right)^{2 / 3}=r \delta(r)
\end{aligned}
$$

Lubricant film thickness:

$$
\delta(r)=\frac{1}{r}\left(\frac{3 Q_{c u} \chi}{2 \pi l \omega R_{0}^{3}}\right)^{2 / 3}
$$

Where: $\delta(\mathrm{r})$ - is the thickness of the boundary layer, $\mathrm{cm}$; $\mathrm{Q}$ - is the flow rate of the mixture; $2 \pi \mathrm{r}$ - is the circumference; $\omega$ - is the angular velocity of the growing particles of the mixture, $\mathrm{m} / \mathrm{s}$; $\mathrm{R} 0$ - is the radius of the speed of the mixture; 1 - is the turbulent.

These expressions lie in the zone from the laminar flow motion to the turbulent zone. Consider the turbulent flow of a propeller, (the propeller is a mixer device) when the Reynolds number Re $>105$

With a strong turbulent flow, the formula of L.A.Satkeevich is used for the mixing length:

$$
l_{r}=\chi \sqrt{z}(\delta(r)-z)
$$

For a second flow rate in the integrated boundary region of the mixture, we obtain the following expression:

$$
Q=\frac{2 \pi \vec{\omega} R_{0}^{3}}{\chi} \hat{r}^{3 / 2} I_{0} \hat{\delta}(r)
$$


In this case, $\mathrm{I} 0=\pi$ calculations show that the surface tensile strength of the fluid in the laminar mode is weaker than in the turbulent mode, which means that the flow around the propeller adversely affects leakage.

To clarify the above, we prepared a laboratory production facility (Fig. 1). Alternatively, a device is provided based on a selective method for removing engine oils from oxidation products. At the same time, the research methodology, engine oil pollution, experimental studies and the effectiveness of its cleaning system, the oil pollution process are private.

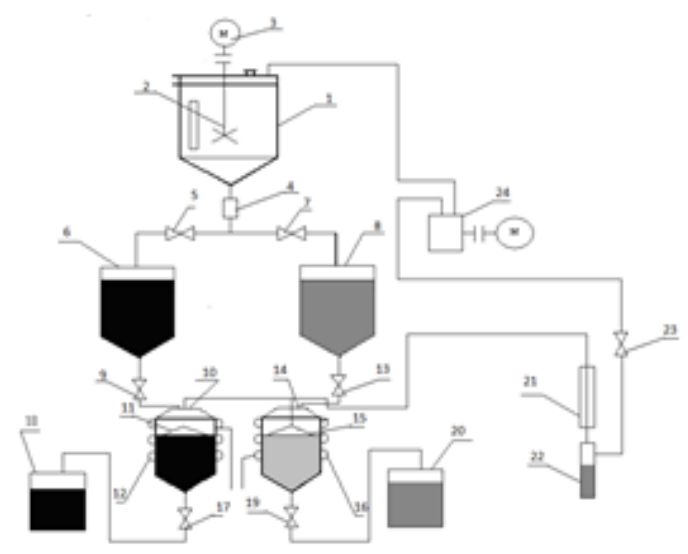

3.

Figure 1. General view of the stand for cleaning motor oils from oxidation products:

1-tank; 2- mixer-settler; 3- engine; 4-viewing window; 5 and 7-taps; 6 and 8 - containers for collecting contaminated and refined oil; 9 and 13 cranes; 10 and 14 - containers for evaporation of the solvent; 11 and 15 - evaporator plates with electric heating; 12 and 16 - spirals; 17 and 19- cranes; 18 and 20 - containers for collecting contaminated and refined oil; 21 - refrigerator; 22 - condensate; 23 - crane; 24- pump.

The laboratory - production unit operates in the following order: the spent oil is poured into the tank together with an acetone solvent; the solution in the tank is mixed with a propeller mixer for 12 minutes, and then settles for 23 minutes. Then, on the basis of the indications of the viewing window, it is separated by taps into containers for cleaned and dirty oils, while acetone is evaporated using evaporation plates, and then it is divided into containers for cleaned and dirty oils.

Laboratory and operational studies conducted using the proposed laboratory - production plant showed a change in the properties of the oil when using selective solvents. A cleaning agent is prepared for the oxidizing oil products used for containers, mixers, disintegrating devices and containers for purified oils and solvents for mixing with an oil-soluble compound.

The following are the results of the selective deletion process. It has been shown that oil refining studies, when the temperature of the mixture is $180 \mathrm{C}$, the effectiveness of acetone and a variety of base oils are improved by increasing the acetone content in the oil. The maximum cleaning efficiency is expected to be 35 to 40 minutes for the mixture. If the ratio of acetone to oil is $80 \%, 60 \%$ and $40 \%$, cooling can be achieved within 35 minutes. Cooling can also be achieved within 40 minutes if the ratio of acetone to oil is $80 \%, 60 \%$ and $40 \%$.

Most authors believe $[10,11,12]$ that a mixed dirty oil in a container should have a limited speed, since a high speed of movement of the hydrodynamic point of view is disadvantageous.

In addition, although it leads to an increase in the intensity of heat transfer, it also increases the energy consumption for mixing. This can be easily verified by analyzing the well-known equations of heat transfer and hydraulic resistances during turbulent fluid motion in a tube heat exchanger [13]

$$
\begin{aligned}
\mathrm{N}_{u} & =0,021 R_{e}^{0.8} \times P_{z}^{0.43} \\
\Delta P & =\frac{\xi_{m p} \ell}{d}+\frac{\sum \xi_{\mu} \rho \omega^{2}}{2}
\end{aligned}
$$

Where: $\mathrm{Nu}$ - is the Nusselt criterion; $\mathrm{R}_{\mathrm{e}}$ - Reynolds criterion; $\mathrm{P}_{2}$ - Prandtl criterion; $\Delta \mathrm{P}$ - is the hydraulic resistance of the heat exchanger on the side of this working medium; $\omega$ - is the speed of the working medium; $\rho$ - is the density of the medium; e - is the length of the channel; $d$ - is the channel diameter; $\xi$ - is the coefficient of friction; $\Sigma \xi_{\mathrm{M}}$ the sum of the coefficients of local resistance.

From (8) and (9) we find $\alpha=\mathrm{A}_{1} \omega^{0.8}, \Delta \rho=\mathrm{A}_{2} \omega^{1.75}$

where: $\mathrm{A} 1$ and $\mathrm{A} 2$ are the proportionality coefficients. From the obtained relations it is seen that a 2 -fold increase in the flow rate ensures a 1.75 -fold increase in heat transfer, and an increase in hydraulic resistance in this case up to 3.4 - fold..

From the above considerations, it follows that it is impossible to consider the intensification of the process in isolation from the energy expenditures produced in this case. $[14 ; 15]$.

According to this experiment to identify the cleaning performance depending on the cooling time in different proportions of acetone and oil, the following ratio was obtained (Figure 2.). Studies on the cooling of the oil used in various proportions of acetone and engine oil when used to heat a mixture with a temperature of $270 \mathrm{C}$ showed that the efficiency of oil purification improves with the acetone content in the oil.

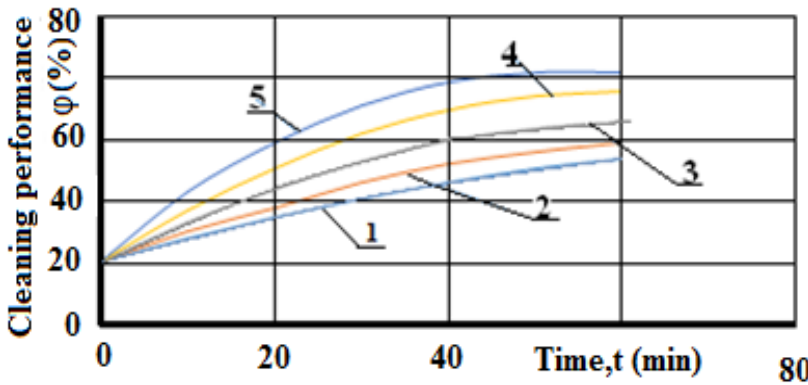

Figure 2. Graphs of cleaning efficiency $(T=270 C)$ depending on cooling time in various proportions of acetone and oil: 1-acetone $80 \%+$ oil $20 \%$, 2-acetone $60 \%$ + oil 40\%, 3-acetone 50\% + oil 50\%, 4-acetone $40 \%$ + oil $60 \%, 5$-acetone $20 \%$ + oil $80 \%$ 
The graphs shown in Figure 1 correspond to the following regression equations:

1 -acetone $80 \%+$ oil $20 \%$, method: $\varphi=-0.005 \mathrm{t} \wedge 2+$ $0.914 \mathrm{t}+18.81$

2-acetone $60 \%+$ oil $40 \%$, method: $\varphi=-0.007 \mathrm{t} \wedge 2+$ $1.15 \mathrm{t}+18.85$

3-acetone $50 \%+$ oil $50 \%$, method: $\varphi=-0.012 \mathrm{t} \wedge 2+$ $1.525 \mathrm{t}+18.59$

4-acetone $40 \%+$ oil $60 \%$, method: $\varphi=-0.017 \mathrm{t} \wedge 2+$ $1.975 \mathrm{t}+18.59$

5-acetone $20 \%+$ oil $80 \%$, method: $\varphi=-0.022 \mathrm{t} \wedge 2+$ $2.405 t+19.25$.

If the ratio of acetone to oil is $80 \%, 60 \%$ and $40 \%$, cooling can be achieved within 25 minutes.

Also, cooling can be achieved within 30 minutes if the ratio of acetone to oil is $80 \%, 60 \%$ and $40 \%$.

The maximum cleaning effect is $20-25$ minutes of cooling, if the temperature of the mixture is 500C. Also, cooling can be achieved within 20 minutes if the ratio of acetone to oil is $80 \%, 60 \%$ and $40 \%$.

With the exception of the clarity of the refined oil, all indicators are in compliance. One of the main reasons for the low transparency of cooled refined oils is the difficulty in separating strongly bound organic matter in the oil. It is advisable to mix it with a long-term solution to remove all organic substances from the oil. According to many scientists, this indicator does not significantly affect the engine friction process.

Table 1. Comparison of physical and chemical properties of used and refined oils \& Results

\begin{tabular}{|c|c|c|c|c|}
\hline № & Title & Used oil & $\begin{array}{c}\text { Refined Oil } \\
(50: 50)\end{array}$ & $\begin{array}{c}\text { According to } \\
\text { state standard } \\
\text { specification }\end{array}$ \\
\hline 1 & $\begin{array}{c}\text { The composition of the } \\
\text { mechanical mixture, } \%\end{array}$ & 0,45105 & 0,014 & 0,015 \\
\hline 2 & $\begin{array}{c}\text { Kinematic viscosity, } \\
\mathrm{mm} 2 / \mathrm{s}\end{array}$ & 9,9 & 8,92 & 8,0 \\
\hline 3 & $\begin{array}{c}\text { Amount of acids, mg } \\
\mathrm{KOH} / \mathrm{g}\end{array}$ & 6,13 & 1,65 & 2,0 \\
\hline 4 & $\begin{array}{c}\text { The amount of alkali, mg } \\
\mathrm{KOH} / \mathrm{g}\end{array}$ & 2 & 4,7 & 6,5 \\
\hline 5 & \multicolumn{2}{|c|}{ Color } & Above 8 & Above 3 \\
\hline
\end{tabular}

The numbers in table 1, confirm the effect of the engine oil cleaner. To optimize the device settings, a three-stage plan of the second level Boxing-Benkin plan was implemented. The total number of points in the plan is 27 , and the number of changing points is - 4 . The calculations were carried out on an electronic machine IBM PC XT.

The regression equation has the following form:

$y=3,1+0,82 X_{1}+1,85 X_{2}+1,96 X_{3^{-}}$

$0,44 X_{4}+2,42 X_{1} X_{2}+0,86 X_{1} X_{3}-0,64 X_{1} X_{4}+3,01 X_{2} X_{3}-0,76 X_{2} X_{4}^{-}$

$-0,21 X_{3} X_{4}+0,71 X_{1}^{2}+0,38 X_{2}^{2}-1,6 X_{3}^{2}-1,5 X_{4}^{2}$

Here are the encoded factors X1, X2, X3, X4 (respectively, the temperature of the solution, the duration of cooling, the ratio of acetone and oil, the concentration of pollution). The graphical method was used to find conditional extremer. [16;17]

\section{RESULTS}

Thus, the best flowering result for oil - $\mathrm{P}(\mathrm{P}=2.4)$ was $5000 \mathrm{C}$, the cooling time was 60 minutes, the coefficient of the mixture was reduced to 50:50, and the concentration of pollution was $0.22 \%$. With a high concentration of mechanical compositions, the color of the oil disappears with increasing oil temperature. From a physical point of view, this can be explained by the fact that, with an increase in the temperature of the solution, the light fractions of acetone evaporate, which, in turn, reduces the capacity of the acetone resin.

An increase in temperature has a positive effect on cooling. At $\mathrm{T}=500 \mathrm{C}$, when the cooling time is 23 minutes, and the concentration of mechanical mixtures is 50:50, and the ratio $\mathrm{X}=0.08-0.15 \%$, the color of the oil is $\mathrm{R}=2.4$.

Thus, a high oil color can be obtained in the following operating modes:

- The ratio of the mixture by volume 50:50;

- Oil temperature 500C;

- cooling time 23 minutes;

- The concentration of mechanical mixtures of 0.08 $0.15 \%$.

In experiments, filters were periodically regenerated and retested after each resource cycle. The content of organic pollution was determined by the acidity and acid content.

Studies have shown that the mass contamination of refined oil decreased by about 3-4 times the amount of contaminants in the oil sample.

For the experiments, $1.2 \%$ oil containing most of the organic ingredients was selected. In this case, the flash point was $1650 \mathrm{C}$, and the kinematic viscosity was: $6.82 \mathrm{~mm} 2 / \mathrm{s}$ at $1000 \mathrm{C}$. As shown in table 3, the refined oil parameters meet standard requirements.

\section{CONCLUSION}

Thus, the following conclusions can be made: - motor oils are intensively contaminated with oxidation products during processing, thereby accelerating the friction process. During agricultural work, tractor engines due to congestion and oxidation of carbon, the content of carbine, carbide and asphalt products increases by $1.2 \%$. Oxidizing products accelerate the deterioration of the quality of oils, which leads to an increase in the power consumption of the engine $\mathrm{KShM}$ and CPG by $15 \%$. Analytical relationships are obtained representing the movement of a mixture of acetone and used motor oil in viscosity states and the process of evaporation of the solvent from the oil during processing. The speed of motor oil on a cone-shaped surface is substantiated based on its effect on refining, and the dependence of the quality of refined oil on the temperature of the mixture and the availability of the evaporation process is justified.

\section{REFERENCES}

1. Sh.Zh.Imomov. Engineering design calculation of a biogas unit recuperator. Applied Solar Energy, 2007, 43(3), pp. 196-197.

2. Sh.Zh.Imomov. Heat transfer process during phase backand-forth motion with biomass pulse loading. Applied Solar Energy, 2009, 45(2), pp. 116-119.

3. Sh.Imomov Technological foundations of the recuperation of heat wastes in biogas installations. ?ashkent, 2011. $136 \mathrm{p}$. 
4. K.A.Sharipov, N.A.Holikova. Optimization installation performance for nanofiltration technics. AGRO-ILM. Tashkent, 2009. ?3(10). pp.56.

5. P V.N.Rokopyev, R.I.Sinyanskaya, E.V. Mishchenko. The method of regeneration of used lubricating oil. Pat. 2076898 Russia, MPK6S 10 M 175/02. 93026982/04.3.

6. A.E. Monastic. Regeneration, drying and degassing of transformer oil. St. Petersburg: PEIPK, 2002, 40 p.

7. A.IO.Evdokimov, A.Yu.Evdokimov, I.G.Fuchs, T.N.Shabalina, L.N.Bagdasarov. Lubricants and problems of chemotology. Russia, 2000. 424 p.

8. M.V.Seleznev, A.A.Mainntsev. Dynamics of changes in the density of gear oils in the conditions of operation of KamAZ automobiles. Theoretical and applied aspects of modern science. 2014, ?2-1. p.83-85.

9. L.R.Rudnik. Additives to lubricants. Properties and application: "Profession", 2013. 928 p

10. K.A.Sharipov, N.A.Halikova. Optimization Installation performance for nanofiltration technics. AGRO ILM. Tashkent, 2009, ?3(10), pp.56

11. N. Khalikova, O. Mukhiddinov. Membrane plants refined oil. Sustainable Agriculture ?1 (1) 2018 ???, 2128 pages.

12. K.A.Sharipov, N.A.Halikova. Establishing patterns of flow of a viscous fluid on the lateral surface of the rotating cone. European science review, ? 1-2 2018 ??? 260-262 pages. 